\title{
PLM implementation guidelines - relevance and application in practice: a discussion of findings from a retrospective case study
}

\section{Mattias Bokinge* and Johan Malmqvist}

Department of Product and Production Development, Chalmers University of Technology, SE-412 96 Gothenburg, Sweden E-mail: mattias.bokinge@chalmers.se E-mail: johan.malmqvist@chalmers.se *Corresponding author

\begin{abstract}
In this paper, a recently conducted product lifecycle management (PLM) implementation project is analysed. The aims are to investigate whether published product lifecycle management (PLM) implementation guidelines are relevant to and used in practice, and, if so, to assess how useful they are for guiding project execution. This paper presents an examination of how a real PLM implementation project was conducted, mapping out the rationale for different courses of action and the effects they had. This paper evaluates the degree of relevance and application of existing PLM implementation guidelines. It is found that while most of the guidelines were highly relevant to the project, they were not applied in full. Potential reasons for why the guidelines are not followed are discussed. It is suggested that projects review their plans with the guidelines in mind, evaluating their degree of relevance and including a plan for how to apply the guidelines.
\end{abstract}

Keywords: PLM implementation; product data management; PDM; empirical study; guidelines.

Reference to this paper should be made as follows: Bokinge, M. and Malmqvist, J. (2012) 'PLM implementation guidelines - relevance and application in practice: a discussion of findings from a retrospective case study', Int. J. Product Lifecycle Management, Vol. 6, No. 1, pp.79-98.

Biographical notes: Mattias Bokinge is a $\mathrm{PhD}$ student in the research group 'Systems Engineering and PLM' at Chalmers University of Technology, where he obtained his MSc in Mechanical Engineering in 2009. His research interest is implementation of PLM solutions.

Johan Malmqvist is a Chair Professor in Product Development at Chalmers University of Technology. His research focuses on development methodologies and IT support for product development (PLM). The research is conducted in close collaboration with Swedish industry. His current projects investigate methods and tools for the development of product-service systems and knowledge-based engineering tools. 


\section{Introduction}

\subsection{PLM implementation - motives and challenges}

The strategic approach of product lifecycle management (PLM) has emerged to support the information flow in product realisation-related processes. According to Stark (2004, p.2), "PLM is the activity of managing a company's products all the way across their lifecycles in the most efficient way. In doing so, it enables the company to take control of its products". The concept of PLM is enabled by a PLM solution, which is a combination of business processes, methods, engineering applications (such as CAD, CAM and CAE) and product data management (PDM) systems. PDM systems provide support for a combination of electronic data vaulting, document management, product structure management, process and project management, and component classification (CIMdata, 2002).

Several vendors of engineering applications and PDM systems, such as Dassault Systemes and Siemens, continuously expand their offer to include functionality to support more phases of the product lifecycle. However, they often fail to make a distinction between the concept of PLM and the applications and systems to support the PLM concept (Schuh et al., 2008). In reality, only a part of the available functionality of each application and system may be utilised by the company, and several different applications and systems from multiple vendors together with business processes and methods combine a company's total PLM solution.

In this paper, we define PLM implementation as the activity of moving from current state to future state regarding the PLM solution and the organisation using it.

When using a commercial PLM system in a PLM implementation project, a gap always exists between the desired processes and the available support from the system. Therefore, two main strategies exist in PLM implementation projects: either adopt the commercial system to fit the desired processes or change the desired processes to fit the existing support in the commercial system (Saaksvouri and Immonen, 2005).

The economic benefits of more efficient PLM solutions are well-known. However, other benefits may be highlighted as well. More efficient PLM solutions may reduce the environmental load occurring in the development process (for example, in less $\mathrm{CO}_{2}$ emissions from travel to meetings and less material consumed to produce physical prototypes). Moreover, like other major organisational changes, PLM implementation projects add to the already existing pressure in organisations. Smoother transitions from current state to future state minimise the extra pressure, thereby contributing to a healthier work environment.

PLM implementation projects are complex. Grönvall (2009) compares PLM implementation with heart transplantation. He also states that PLM implementations carry many dependencies and uncertainties, and are therefore high-risk projects. Several authors (for example, Saaksvouri and Immonen, ibid) stress the importance of a thorough analysis of business processes and requirements before implementing PLM. Some also stress that while the purely technical part in itself might be a challenge, the organisational part is even harder (for example, Garetti et al., 2005). Hewett (2009) identifies three such issues: cultural-related issues regarding the product engineers, issues regarding immature PDM systems, and non-standardised engineering processes. 


\subsection{Existing PLM implementation support}

To direct PLM implementation projects, several sets of guidelines have been presented in literature. In this paper, we define a guideline as a directional recommendation for what to do (or what not to do) in a specific context. In the following section, we summarise available PLM implementation guidelines.

Pikosz et al. (1997) discuss introduction strategies for PDM systems and present lessons learned based from four case studies in Swedish industry. They recommend conducting a pre-study before system selection, securing benefits for all stakeholders, establishing user involvement and top management support, improving processes before or simultaneously with the project, and performing a pilot study before doing a full implementation. Rangan et al. (2005) focus on organisational aspects in PLM implementations and refer to their own field experience, as well as to several published case studies (for example, Jennings and Rangan, 2004; Illback and Sholberg, 2000; Chadha and Welsh, 2000). They recommend aligning processes with system capabilities; dividing the project into sub-projects; and allowing different solutions for different parts of an organisation. Grieves (2006) also emphasises organisational change management in PLM implementations and refers to lessons learned from the ERP domain, based on a study of different ERP implementations performed by Brown and Vessey (2003). They emphasise the importance of management engagement, authorised project participants, the use of third party expertise, change management, and satisfying rather than optimising. Wognum and Kerssens-van Drongelen (2005) present lessons learned about the selection and implementation of PDM system functionality, also focusing on organisational aspects, based on a survey of eight Dutch companies. They recommend carefully planning the project, adjusting to business changes, securing that users have a collaborative attitude, and educating the users properly. Hartman and Miller (2006) advance lessons learned about the selection and implementation of PDM and PLM-related technologies, based on interviews with management staff from ten US companies. They recommend the alignment of the system with business processes, to have upper management support, to minimise the amount of customisations, and to educate the users properly. Meanwhile, Berle (2006) presents lessons learned from his own experience of a PLM effort and recommends not underestimating the magnitude of the change; ensuring management support; providing a business case that can be understood by end users; dividing the project into sub-projects; implementing only existing software; involving stakeholders from cross functional divisions; and developing processes in connection with the project. Zimmerman (2008) summarises findings from a study of a more than ten-year long PLM implementation project. In order to ease future projects, he recommends controlling project progression, dividing the project into sub-projects, and establishing a coherent multi-layered PLM architecture. A summary of the above guidelines is presented in Table 1. A more detailed description of the above PLM implementation guidelines has been compiled by Bokinge (2011).

The guidelines above span the complete process, from the establishment of coherent PLM architecture (in order to ease future projects) to the proper education of users. However, a common theme is that they state what needs to be accomplished, but provide less guidance as to how to carry out the task (how to align processes with system capabilities, for example). In addition, where cases are referred to, the implementations as such are only briefly described [with the exception of Zimmerman (2008)]. As a result, readers of those articles may find it difficult to understand the rationale behind various 
guidelines to be applied in practice and what the consequences can be if they are not applied.

Table 1 Summary of published PLM implementation guidelines

\begin{tabular}{ll}
\hline Guideline category & Guideline \\
\hline Project process & Divide project into sub-projects \\
& Perform a pilot project \\
& Conduct pre-study prior to system selection \\
& Plan carefully \\
& Follow-up and control project process \\
& Be prepared to adjust the plan when business changes \\
& Define benefits for all stakeholders \\
& Aim to satisfy rather than optimise \\
& Do not force the same solution on the whole organisation \\
Goals & Carefully estimate the magnitude of change \\
& Establish a coherent PLM architecture \\
& Improve processes prior to or simultaneously with PLM projects \\
& Align processes with system capabilities \\
Only roll out tried software releases & Minimise customisation \\
Enstem and process design management support & Involve users from all departments and disciplines \\
Authorise the project participants & Use expertise from third parties \\
Educate system users
\end{tabular}

Several reference process models for PLM implementation have also been proposed in literature (for example, Schuh et al., 2008; Bitzer et al., 2008; Batenburg et al., 2006; Kumar and Midha, 2006). They mainly focus on support for early phases of a PLM implementation project, which may result in a system being selected. However, they provide fewer detailed instructions for subsequent tasks (how to customise the system, for example). Other authors compare or recommend the use of different implementation processes (for example, Morandotti, 2007; Wognum and Kerssens-van Drongelen, 2005). The recommendations thus differ; no dominant PLM implementation reference process has yet emerged.

We conclude that there is a lack of research on PLM implementation guidelines that focuses on the operational level. More specifically, there is a lack of studies that examine what guidelines are relevant for and used in real industrial PLM implementations, why (or why not) they are used, and what their value is if applied. Rangan et al. (2005) argue that the main body of knowledge of how to implement PLM resides in the heads of individuals who lack the incentives to share their experiences. We argue that systematic studies of real implementation efforts are essential in order to bring out and codify this knowledge. 


\subsection{Research aim}

In this paper, we aim to compare current practice in a conducted PLM implementation project with the PLM implementation guidelines summarised above. More specifically, we aim to thoroughly describe the project, assess the degree of relevance and application of each guideline, and discuss potential reasons for why relevant guidelines are not used in industry.

\subsection{Paper outline}

The remainder of this paper is structured in the following way. Section 2 first outlines the research approach taken in the study. A thorough description of the project studied is then presented in Section 3, followed by a comparison between that project and the PLM implementation guidelines in Section 4. We then discuss the research approach and the usability of the results in Section 5. Finally, we present our conclusions in Section 6.

\section{Research approach}

PLM implementation projects are complex and multi-dimensional. Project organisation, process, methods, and changes in the global economy are only some of the aspects that affect project conduct outcome. Therefore, a qualitative systems approach has been used in the research. The approach calls for an in-depth case study (Yin, 2003). Multiple data sources were utilised, such as interviews, documents, reference group meetings with company employees, and seminars, in order to understand the underlying factors for courses of actions and minimise bias. In the following sections, we describe the empirical setting, and the data collection and analysis approach, respectively.

\subsection{Empirical setting}

The project studied was conducted during the period 2006 to 2009 at a multi-national company in the manufacturing industry (hereafter called GlobalGroup). GlobalGroup delivers commercial solutions in various areas, and is divided into multiple divisions, some of which were involved with the studied project (denoted as AlfaDivision, BetaDivision, and GammaDivision in Table 2).

GlobalGroup used (and still uses) several engineering applications and PDM systems with different functionality in their PLM solution. Some applications are developed in-house while others are based on commercial off-the-shelf (COTS) applications and systems from different vendors. Earlier, GlobalGroup had gone through a larger PLM initiative (cf. Zimmerman, 2008) that resulted in a decision to phase out a PDM system from one of their vendors (hereafter called VendorCorp). GlobalGroup decreased the maintenance budget for the system. Nonetheless, the system continued to work well for several years and was not shut down. However, in the summer of 2006, VendorCorp announced that their system support would end in two years' time. Unwilling to take the risk of using a system not supported by the vendor, GlobalGroup decided to replace it, together with some engineering applications and other PDM systems connected to it. However, the PLM solution of their new preferred supplier was not assessed as mature 
enough. As a result, the company decided to replace the old PDM systems and engineering applications with new ones from VendorCorp. The functional scope of the project covered data vaulting, document management, digital mock-up and process support for release management, meaning that the project mainly covered basic PDM system functionality with some engineering applications. A simplified picture of the PLM architecture prior to and after the project is presented in Figure 1.

Figure 1 Simplified basic PLM architecture (a) prior to and (b) after the PLM implementation project (see online version for colours)

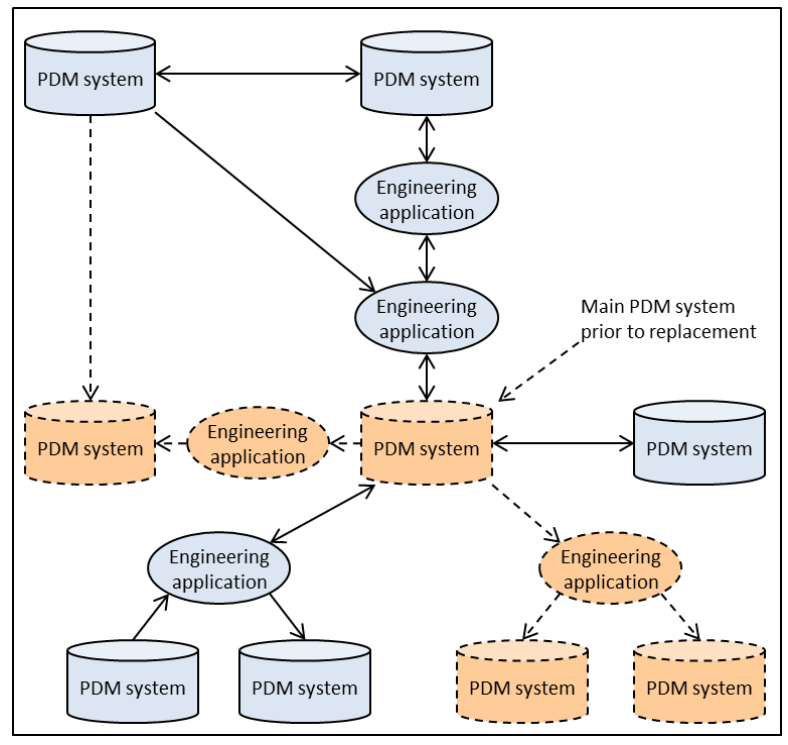

(a)

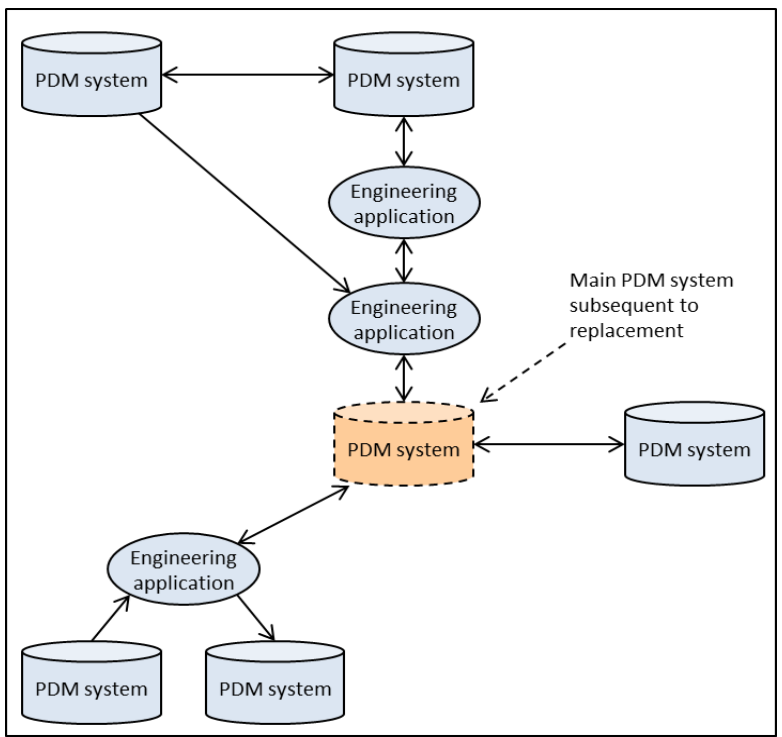

(b) 
Table 2 Interview sample

\begin{tabular}{|c|c|c|c|c|}
\hline & AlfaDivision & BetaDivision & GammaDivision & ITDivision \\
\hline Steering committee members & 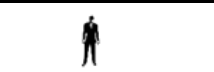 & in & & \\
\hline \multicolumn{4}{|l|}{ IR reference group members } & in \\
\hline $\begin{array}{l}\text { Business reference group } \\
\text { members }\end{array}$ & 1 & 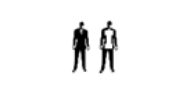 & 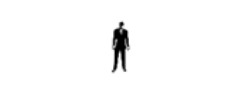 & \\
\hline Project managers & & & & 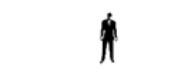 \\
\hline IT project members & & & & 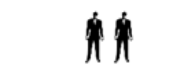 \\
\hline $\begin{array}{l}\text { Middle management and end } \\
\text { users }\end{array}$ & 1 & 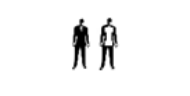 & & \\
\hline \multirow[t]{2}{*}{ Not project participants } & & & & in \\
\hline & GlobalGroup & Veno & orCorp & Consultants \\
\hline Steering committee members & 1 & & & \\
\hline \multicolumn{5}{|l|}{ IR reference group members } \\
\hline $\begin{array}{l}\text { Business reference group } \\
\text { members }\end{array}$ & & & & h⿱㇒日 \\
\hline Project managers & & & & $i$ \\
\hline IT project members & & & & $1 h$ \\
\hline $\begin{array}{l}\text { Middle management and end } \\
\text { users }\end{array}$ & & & & \\
\hline Not project participants & & & & \\
\hline
\end{tabular}

Note: $\hat{h}=$ accounted for in one additional role

The project was performed in a multi-national environment in a company with multiple sites and company divisions around the world. It allows for insights into a wide range of PLM implementation issues, such as changes to the organisation, business processes and methods, and changes to the PLM solution itself. Although the two PLM solutions were developed by the same vendor, they are significantly different. The architecture and user interfaces of the solutions differ, and the new solution enables a much more comprehensive PLM support.

The company studied had performed several PLM implementation projects prior to the actual case. Therefore, we argue that the project represents current practice within the field, without having to regard 'beginner' issues. Also, the project recently ended, during the fall of 2009. Therefore, the findings reflect current PLM implementation practice.

\subsection{Data collection}

Seventeen semi-structured interviews (with 21 interviewees) were performed during the case study, varying between one to three hours each. The interviewees were sampled 
according to a purposive heterogeneous strategy in order to represent as many viewpoints as possible (see Table 2). One interview was done with two interviewees from the company's requirement engineering methods department, in order to analyse similarities and differences between the studied case and the company's abilities in general. All interviews were based on an interview guide, with roughly 40 questions covering the interviewee's background, the implementation process and requirement engineering methods, the project organisation, and project outcomes. The researchers' knowledge of the implementation project grew with the carrying-out of each interview, which in turn allowed for deeper questions and answers. All interviews were done by at least two interviewees, and were recorded, transcribed, and sent to the interviewees for validation.

In addition, more than 200 project and company documents were analysed. Examples of those documents include white books, meeting minutes, communication letters, and technical documentation.

\subsection{Data analysis, validation, and synthesis}

Interview statements were categorised into 26 analysis areas and grouped into five main areas. Communication letters and meeting protocols were summarised in a few sentences and added to the analysis material, and the technical project documentation was analysed in depth.

For validation, preliminary findings have on three occasions throughout the study been presented for a reference group. The group consisted of managers from the IT department and other departments. A presentation with final findings was held for the reference group and most of the participating interviewees. In addition, two presentations were held at another company, with characteristics similar to GlobalGroup. All of these groups corroborated the validity of the findings.

Finally, a synthesis activity was undertaken, where the findings were compared with available empirical based support from literature, the earlier mentioned implementation guidelines. Recommendations were generated for future projects by extending the published implementation guidelines with new ones and proposing a review of PLM implementation project plans with the guidelines in mind.

\section{Project description}

In this section, a description of the studied project is provided, including the implementation process and the project evaluation.

\subsection{Implementation process}

The project followed GlobalGroup's global project model for the development of information systems. It is a waterfall stage-gate model with seven phases (pre-study, concept study, development, final development, validation, deployment, and follow-up) (see Figure 2). In this section, a chain of events through the different phases is presented. 
Figure 2 GlobalGroup's project model for the development of information systems (see online version for colours)

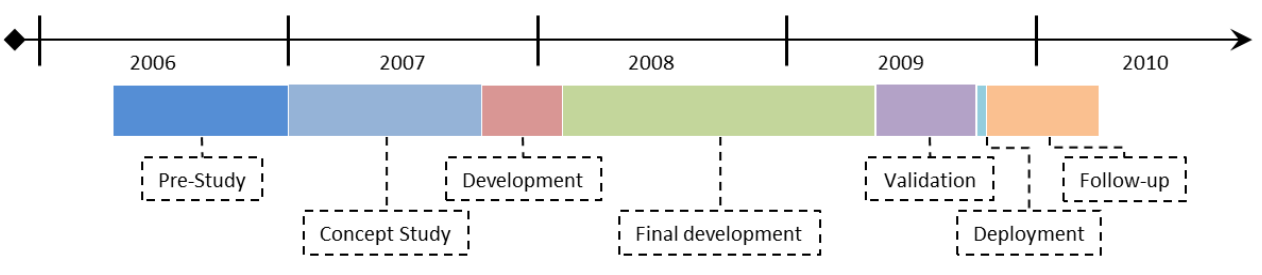

\subsubsection{Pre-study phase [October 2006 to January 2007]}

A pre-study team, led by the business divisions with input from the ITDivision, concluded that the most suitable solution would be to replace the existing PLM solution with a new one from VendorCorp, despite GlobalGroup's strategy to phase out the use of VendorCorp's PDM systems. VendorCorp provided GlobalGroup with contacts to other corporations that had recently conducted similar projects to learn from their experience.

A main project objective was to minimise risk, since using a solution not supported by the vendor would have been a huge business risk. Another objective was to perform the replacement rather quickly, since the vendor initially announced that the support would end in the summer of 2008. Also, GlobalGroup considered the existing PLM solution to be a phase-out system; therefore, a third project goal was to minimise the cost of the implementation.

To minimise time and cost, processes were to remain unchanged. The project aimed at a ' $1: 1$ replacement' of the existing PLM solution with a particular release of VendorCorp's new generation PLM solution. The ratio 1:1 meant that all current processes should be supported by the new solution. However, many stakeholders interpreted 1:1 as having the exact same functionality in the new solution as in the existing one. GlobalGroup planned to launch the new solution during the fall of 2008, and VendorCorp agreed to prolong the support of the existing solution until the new one had been launched.

The project had difficulties getting commitment from all necessary divisions. However, it was ultimately given the go-ahead, and a project organisation was setup.

\subsubsection{Concept study phase [January 2007 to November 2007]}

In beginning of the concept study phase, it became evident that preliminary cost estimations were too low. Several estimations had previously been too optimistic, and a budget for some necessary areas was missing. Since the project was supposed to be a 1:1 replacement, the business divisions thought there was no need for them to get involved. They argued that the ITDivision should know how the old solution was being used. However, the lack of an adequate maintenance budget for several years had resulted in insufficient documentation of the existing solution. Therefore, documentation for how the existing solution worked had to be created as part of the project. This led to additional project costs not previously anticipated. New calculations performed by the ITDivision pointed to twice the first estimated amount.

The ITDivision elicited requirements and validated them with the business reference group. Project vision, use cases (functional requirements), and supplementary 
requirement specification (non-functional requirements) were constructed. The specifications were based on existing templates created for new development projects. In order to understand the possibilities and constraints of the new solution, the business reference group members participated in a training course in the off-the-shelf version. Nonetheless, business reference group members from different divisions still had difficulties agreeing on the requirements. All divisions used the existing solution in different ways, and it was unclear what functionality could be customised in the new solution for each division and what functionality had to be common. Not all business reference group members were authorised to make decisions. Furthermore, the 1:1 replacement strategy made it difficult to secure benefits for all stakeholders. Benefits existed on a global collaboration layer, but there were fewer benefits for an individual user. The difficulties of specifying the requirements led to an increase in time and cost.

A project audit, led by GlobalGroup representatives, concluded that the various business divisions had to unite their visions and agree upon requirements. It was also suggested that concept prototypes should be constructed in order to identify critical areas where the COTS solution would not be enough.

The ITDivision constructed the concept prototypes (essentially solution mock-ups in presentation slide format) and presented them to the business reference group. Based upon reviews of the concept prototypes, it became evident that the out-of-the-box PDM system release initially aimed at would require major customisations to meet the GlobalGroup's needs. The project sent a change request with additional functionality to VendorCorp, who agreed to include the new functionality in their next release. The project decided to implement the forthcoming release instead of the existing one, and the consequence was a time delay.

A new, large PLM concept initiative had started and was now running simultaneously with the implementation project. VendorCorp was evaluated once more and compared with its competitors by GlobalGroup. This evaluation further delayed the implementation project. However, in late 2007, it was concluded that VendorCorp was one of the two remaining competitors going through a final evaluation (they later won the evaluation). Commitment from business units in the implementation project increased substantially. Shortly after the notice, the project continued to the next phase, bringing with it almost finished use cases, supplementary requirements specifications and concept prototypes.

\subsubsection{Development phase [November 2007 to January 2008]}

However, work had continued while waiting for the result from the PLM concept initiative. Therefore, the development phase was short, lasting from November 2007 to January 2008. The use cases, the supplementary requirement specifications and the concept prototypes were finished and approved. It was now found that the largest project risk would be a delay of the new PDM system release from VendorCorp, who had promised to deliver the new release in July 2008.

\subsubsection{Final development phase [January 2008 to May 2009]}

In the beginning of the final development phase, VendorCorp announced that the targeted PDM system release would be delayed. It was not delivered until November 2008, and the quality of the release was assessed as being insufficient for roll out. Therefore, the project had to wait for a maintenance release delivered in February 2009. 
The IT project tested the solution in two ways, by internal testing and business acceptance tests. Test scenarios were based (but modified) on the concept prototypes. Several runs of tests were performed (from the fall of 2008 to the spring of 2009) that revealed important issues with the solutions, regarding both functionality and performance. Performance was slower than what was expected by the users, especially for large assemblies. The date for deployment was postponed several times, mostly because the project had difficulties progressing. In addition, a business division announced that deployment for them would not be possible at a particular time, due to heavy product releases for one of their departments. The steering committee requested that the business reference group accept the workarounds and changes that the IT project suggested, so that the project could move on. Finally, the deployment date was set for August 2009 .

\subsubsection{Validation phase, deployment phase, and follow-up phase [May 2009 to April 2010]}

The validation phase started in May 2009. The final business acceptance tests were performed, and, after the summer vacation, users were scheduled for training. After a final migration rehearsal, the project progressed to the deployment phase and replaced the solutions in September 2009, about a year later than initially planned. In October 2009, the responsibility for the solution was transferred to the maintenance department. In the follow-up phase, learning lesson documents were compiled by the business project, the IT project and the solution vendor.

\subsection{Project evaluation}

In summarising the project results, some gains can be identified. While replacing the old PDM system, several engineering applications and PDM systems became unnecessary and were removed as well. This led to a less complex architecture with a decreased number of engineering applications and PDM systems. Also, divisions and even departments therein used the existing solution in their own ways, with different methodologies. GlobalGroup now has a globally standardised way of working (with release management, for example) that has been enabled (and enforced) by the new solution.

However, the initial budget was overrun by a factor of three. A too optimistic initial budget assessment is part of the explanation. But a string of events caused additional delays and thus cost overruns. Those events included the following: aiming for the wrong PDM system release, poor quality of the initial version of the target release, delays in internal development, and delays due the fact that the product release was prioritised.

Regarding quality, benefits were gained on the divisional collaboration level through more efficient information sharing. However, end-users indicated a decrease in individual user efficiency. One example of this was functionality that required more and non-intuitive mouse-clicks. In addition, the project consciously disabled a specific functionality, which led to decreased user efficiency. User satisfaction also varied between geographical sites. In addition, poor organisational change management, e.g., communication about the change, lack of education and user support when the solution was replaced, probably reinforced the perception of a decrease in user efficiency in the new system. 
In a recent effort, we identified and discussed the main challenges and critical errors in the case (Bokinge and Malmqvist, 2011). We found that the internal challenges were related to: the project scope and goals, the implementation process model, the requirements elicitation and validation, the solution testing and the user involvement. We argued that the project lacked support for defining an overall scope and business case, stating customisation rather than green-field requirements, connecting use cases and tests, and effectively using the reference group. Consequently, the project drifted from a pro-active, requirements-driven mode to a reactive, test-driven mode. However, the project also demonstrates the importance of events beyond the project's control. External challenges, such as a delay of the target release from the vendor, and the need to adapt the launch to the group's product release schedule, also caused significant delays and cost overruns.

Overall, several of the interviewees categorised the project as neither a success nor a failure. It was perceived as a normal outcome at GlobalGroup. They managed to replace their PLM solution, but the project was unnecessarily long and costly.

\section{Comparison with PLM implementation guidelines}

Let us now return to the literature and compare the project's execution with the implementation guidelines identified in Table 1. In the following sections, we review the relevance and application of each guideline in the researched case. Subsequent, additional guidelines proposed based on the case are reviewed as well. Lastly, potential reasons for the guidelines with high relevance and low application are discussed and countermeasures suggested.

\subsection{Guideline category: project process}

- Divide project into sub-projects; perform a pilot project. The project was not divided into sub-projects, and a pilot project was not performed. Given that the project magnitude was relatively limited, it is not certain that the project complexity would have required a division or pilot.

- Conduct pre-study prior to system selection. A pre-study was made prior to solution selection, but it was assessed as insufficient by several interviewees. The estimated amount of work needed was too optimistic, and the budget for some necessary areas was missing. As a result, the project was approved with an insufficient budget. Furthermore, the scope was continuously debated throughout the project.

- Plan carefully; follow-up and control project process. The work was planned and monitored by the steering committee, according to the group's project model. In particular, the system deployment was thoroughly planned and rehearsed and run without major issues. This most probably saved unnecessary costs for the project. However, although cost was rigorously controlled, the quality of the deliveries was not.

- Be prepared to adjust the plan when business changes. The view of PDM systems from VendorCorp changed, from being a system going to be phased out to a system being part of GlobalGroup's future vision. Still, the project progressed without any 
major modifications in the plan. An adjusted plan and objective could have better prepared GlobalGroup for upcoming future projects.

\subsection{Guideline category: goals}

- Define benefits for all stakeholders. Benefits were defined on an architectural level. However, end users did not perceive any benefits on an individual engineering level. Therefore, it took a long time to get users involved in the project, leading to the late discovery of certain requirements.

- Aim to satisfy rather than optimise. The project used business acceptance tests to verify user scenarios, and development continued until it was secured that the scenarios could be supported. According to interviewees, some of the scenarios required multiple and non-intuitive mouse-clicks, which indicated that the project aimed to satisfy rather than optimise. As a result, project costs probably were kept low at the expense of user efficiency.

- Do not force the same solution on the whole organisation. All divisions were forced to use the same solution. Only minor differentiations by configurations and minor customisations were allowed. Although differentiations could have resulted in a more optimal solution for each division, standardisations provided benefits through collaboration between divisions.

- Carefully estimate the magnitude of change. Initially, the project was positioned as a 1:1 replacement, a database transition with no impact on the business. Though it gradually became evident that business would have to change, the initial aim had already resulted in a budget only sufficient for a 1:1 replacement.

\subsection{Guideline category: system and process design}

- Establish a coherent PLM architecture. The maintenance budget of the existing solution was decreased for several years, resulting in a lack of accurate documentation of the solution. The creation of interface documentation had to be added to the project, increasing costs and lead time.

- Improve processes prior to or simultaneously with PLM projects. The project was positioned as a 1:1 replacement, and there was no budget for process reengineering. Some of the processes changed, while others remained the same. As a result, the project increased in cost by adding unnecessary complex customisations to meet the existing processes.

- Align processes with system capabilities. Some of the processes changed, while others remained the same. Processes were aligned with solution capabilities in some areas but not all. As a result, customisations were needed, which increased time and cost.

- Only roll out tried software releases. The project decided to change scope in the direction of a future PDM system release. Meanwhile, the project did not immediately slow down, but tried to perform activities in advance. VendorCorp delivered the new release late and with insufficient quality. As a result, several 
performed activities had to be repeated when the new release was available, increasing both cost and time.

- Minimise customisation. The project was positioned as a 1:1 replacement, and there was no budget for process reengineering. As a result, the project increased in cost by adding unnecessary complex customisations to meet the existing processes. However, the project avoided some customisations by changing the project aim in the direction of a future PDM system release. VendorCorp included new functionality that otherwise would have been achieved through major customisations.

\subsection{Guideline category: organisation}

- Ensure management support. The project had difficulties receiving management support from all business divisions. Although initially approved and funded, it was not until it was announced that VendorCorp could be part of GlobalGroup's future vision that commitment increased from all business divisions. As a result of the late commitment of managers, initial activities most probably took longer than necessary.

- Involve users from all departments and disciplines. Most of the business reference group members were from the headquarter site. They represented their own views rather than those of the global organisation. Lack of time and compensation can be seen as an explanation, since the activities were performed in addition to regular work. As a result, the solution was less optimal for departments located at other sites.

- $\quad$ Authorise the project participants. Project requirements were approved by the members of the business reference group. Not all members were authorised to make decisions, and those who were not had to get back to their local organisation for consultation before a decision could be made. Since decisions could not be made directly at the meetings, project time increased.

- Use expertise from third parties. The project consulted other companies that had recently conducted similar implementations of systems from VendorCorp. Knowledge gained from these discussions most probably increased the outcome of the project. However, the project did not use expertise from companies specialised in PLM implementations. Some problems encountered could probably have been avoided if the project had made better use of third party expertise.

- Educate system users. As a means of reducing project costs, the money spent on training for users was rather low. Users were taught how to perform tasks in the new solution, but not why. In addition, no comparisons with how tasks were performed in the existing solution took place. As a result, the efficiency dip following the project was longer and deeper than necessary. A super-support team needed to be put in place as a counter-measure.

\subsection{Additional guidelines}

In addition to the guidelines reviewed above, some new guidelines can be constructed and added to the list, based on the experiences from this case. 
- Establish a PLM roadmap. Regarding the project process, GlobalGroup reactively started the project when the PDM system lifespan had almost expired, thereby adding an external time constraint. We suggest that projects establish a PLM roadmap, as this would facilitate considerations of the development plan of vendor software.

- Apply project models for COTS implementation. Also regarding the project process, the project used requirement templates for new systems development. However, implementing COTS systems is different from developing systems from scratch. Hence, we suggest that project models for COTS implementation be applied.

- Align project with PLM strategy. Regarding the project goals, the project had difficulty getting commitment from all necessary divisions, due to the company's earlier strategy of phasing out PDM systems from the vendor. We suggest that projects be aligned with the overall PLM strategy.

- Define clear responsibilities for all project groups. Finally, regarding the organisation, the business reference group received little support from GlobalGroup's project model. As a result, the project had to rely on the abilities of individuals, which in this case was not enough. We suggest that clear responsibilities be defined for all project groups.

A summary of all guidelines is presented in Table 3. In it, each guideline is assessed on a three-graded scale (high, medium and low) regarding its relevance and application to the case.

\subsection{Application of guidelines in the case}

It is apparent that the project in full applied rather few of the relevant PLM implementation guidelines. Of the 24 guidelines above, 20 were assessed as having high relevance for the case. Of these 20 , only one was assessed as being followed to a high extent, five to a medium extent, and 14 to a low extent. Why are PLM implementation guidelines not followed despite their relevance? Roughly speaking, potential reasons may fall into one of the following three categories: lack of awareness of the guidelines; awareness but active decision to not apply the guidelines; and, awareness but failure, despite the intent to apply the guidelines.

In the first category, lack of awareness of guidelines, we assume that knowledge about PLM implementation guidelines is not known to, or at least not understood by, project participants. The relevance of a guideline to a specific case can be difficult to assess. That could be due to a lack of context knowledge related to the guideline. Participants might not even search for guidelines, inside or outside the company. It is also possible that search is conducted, but that guidelines are difficult to find. We reviewed guidelines from multiple empirical based cases in order to summarise the list in Table 1. Examples of guidelines not followed in the project due to a lack of awareness are 'do not underestimate the magnitude of change', 'only roll out tried software releases' and 'apply project models for COTS implementation'.

Awareness is a first step, but it is not enough for guidelines to be followed. The second category is when project participants are aware of and have understood guidelines, but make an active decision to not apply them. Although guidelines are 
assessed as highly relevant, other aims or strategies may contradict them. Also, earlier experience of following a certain guideline may have led to costs that were too high or a lack of visible benefits. Examples of guidelines not followed in the project due to a decision to not apply them are 'use expertise from third parties', 'define benefits for all stakeholders', and 'improve processes prior to or simultaneously with PLM projects'.

Table 3 Restatement of the summary of the published PLM implementation guidelines and additional guidelines drawn from this case, with indications of relevance and application in the researched case

\begin{tabular}{|c|c|c|c|}
\hline Guideline category & Guideline & Relevance & Application \\
\hline \multirow[t]{8}{*}{ Project process } & Divide project into sub-projects & Low & Low \\
\hline & Perform a pilot project & Low & Low \\
\hline & Conduct pre-study prior to system selection & High & Low \\
\hline & Plan carefully & High & Medium \\
\hline & Follow-up and control project process & High & Medium \\
\hline & $\begin{array}{l}\text { Be prepared to adjust the plan when business } \\
\text { changes }\end{array}$ & High & Low \\
\hline & Establish a PLM roadmap & High & Low \\
\hline & $\begin{array}{l}\text { Apply project models for COTS } \\
\text { implementation }\end{array}$ & High & Low \\
\hline \multirow[t]{5}{*}{ Goals } & Define benefits for all stakeholders & High & Low \\
\hline & Aim to satisfy rather than optimise & Medium & High \\
\hline & $\begin{array}{l}\text { Do not force the same solution on the whole } \\
\text { organisation }\end{array}$ & Medium & Low \\
\hline & Carefully estimate the magnitude of change & high & low \\
\hline & Align project with PLM strategy & High & Low \\
\hline \multirow{5}{*}{$\begin{array}{l}\text { System and } \\
\text { process design }\end{array}$} & Establish a coherent PLM architecture & High & Low \\
\hline & $\begin{array}{l}\text { Improve processes prior to or simultaneously } \\
\text { with PLM projects }\end{array}$ & High & Low \\
\hline & Align processes with system capabilities & High & Medium \\
\hline & Only roll out tried software releases & High & Low \\
\hline & Minimise customisation & High & Medium \\
\hline \multirow[t]{6}{*}{ Organisation } & Ensure management support & High & Medium \\
\hline & $\begin{array}{l}\text { Involve users from all departments and } \\
\text { disciplines }\end{array}$ & High & Low \\
\hline & Authorise the project participants & High & Low \\
\hline & Use expertise from third parties & High & Medium \\
\hline & Educate system users & High & Low \\
\hline & $\begin{array}{l}\text { Define clear responsibilities for all project } \\
\text { groups }\end{array}$ & High & Low \\
\hline
\end{tabular}

In the last category, we find those guidelines that a project fails to follow despite the intent to do so. In connection with the first category, it is possible that a guideline is discovered too late in order to establish necessary counter-measures. Furthermore, adequate know-how might have been missing, as a result of insufficient resources or 
support from upper management. Examples of guidelines not followed in the project due to a failure to apply are 'minimise customisation', 'follow-up and control project process' and 'involve users from all departments and disciplines'.

We argue that guidelines assessed as having a combination of high relevance and low application are most problematic and need to be analysed in order to achieve better project outcomes regarding time, cost and quality. In the case described in this paper, the project outcomes would most probably have been better, if more of the relevant guidelines had been followed to a better extent. Therefore, we suggest that projects review their project plans with PLM implementation guidelines in mind. The list in Table 3 can be used as a foundation, but more guidelines should be searched for, for example, in literature, at seminars, and from experts. Furthermore, we suggest that the relevance of each guideline be assessed and that plans for how to follow relevant guidelines be constructed.

\section{Discussion of research approach and the usability of the results}

In this paper, qualitative findings from a multi-dimensional context are presented. This paper contributes empirical experiences from a PLM implementation case, based on interviews and project and company documents.

Transferability is an important evaluation criterion in single case studies (Guba and Lincoln, 1989). In this case, that means that the findings put forward can be transferred to the reader of this paper. Our intention has been to facilitate transferability by presenting a thorough description of the project, with rationale for courses of action, prior to the comparison with existing guidelines.

The interviews were performed about half a year after the project ended. Some interview questions concerned activities and courses of action that appeared more than three years earlier. It has been a challenge to grasp the experience and knowledge created by the interviewees through the project. The findings have been presented for our business reference group at GlobalGroup and for interviewees that participated in the study. These presentations are part of this paper's construct validity (cf. Yin, 2003).

The suitability of the empirical setting is also important. We recall that the project was performed in a multi-national environment in a company with multiple sites and company divisions around the world and that it initially was positioned as a replacement project. The scope of the project mainly covered basic PDM system functionality and some engineering applications. If the project had been positioned as an improvement project instead of a replacement project, there might had been decision to follow several of the guidelines (for example, to improve processes prior to or simultaneously with PLM projects, or to align processes with system capabilities). Furthermore, it probably also would be more difficult to define benefits for all stakeholders and to not force the same solution on the whole organisation. However, the case still allows for insights into a wide range of PLM implementation issues, and we argue that it is suitable for validating the relevance of the PLM implementation guidelines.

The study relies on guidelines presented in the PLM context and studies a PLM context. However, one could ask oneself if the guidelines can be generalised to the wider context of IT implementations. The relevance of a guideline to a specific context depends upon the characteristics of that context. Recall that PLM implementations can be 
characterised as complex and high risk endeavours (Grönvall, 2009), where the PLM solution is commonly being based on immature PDM systems and non-standardised engineering processes (Hewett, 2009), and where the solution subsequently is being used by product engineers. Although their importance might vary from case to case, it is reasonable to believe that some of the guidelines can be generalised to projects in general (for example, plan carefully, and follow-up and control project process), some to IT implementations (for example, conduct pre-study prior to system selection, and minimise customisation) and some to IT implementations with for example high complexity and risk (for example, divide project into sub-projects, or only roll out tried software releases). However, not fully understanding the common set of characteristics of PLM and IT in general, we are cautious in making such claims. According to Svensson et al. (2002), the generalisation of findings in qualitative, single case studies is possible through recognition of the results. Hence, readers of this paper, with insights from other IT implementations, might recognise the results herein.

Our suggestion above implies that better application of relevant PLM implementation guidelines leads to better project outcomes regarding time, cost and quality. Comparisons with other cases are needed in order to provide evidence for or against the hypothesis.

\section{Conclusions and future work}

Existing PLM literature proposes many guidelines for what to consider when implementing PLM. However, the guidelines lack concretisation in term of their rationale and what the consequences can be if they are not applied. This paper has contributed to the body of knowledge by summarising PLM implementation guidelines based on empirical case studies and comparing those with a recent case from industry. This paper presents an in-depth, comprehensive description of how a PLM implementation project has been conducted, what the rationale have been for different courses of actions, and what effects they have had. Furthermore, this paper contributes an assessment and discussion of the degree of relevance and application of the guidelines in the case, and potential reasons for the low application of highly relevant guidelines have also been discussed.

It was found that many of the proposed guidelines were relevant to the case, but that relatively few were followed throughout the project. Also, in addition to underline existing PLM implementation guidelines, the case served to identify four additional guidelines.

Three categories of potential reasons for why guidelines were not followed are: lack of awareness; awareness but decision to not apply; and awareness but failure to apply. It is argued that guidelines with a combination of high relevance and low application are most problematic and need to be analysed in order to achieve better project outcomes. In addition, it is suggested that projects review their plans with the guidelines in mind, assessing the degree of relevance and including a plan for how to apply the guidelines.

We recall that the main body of knowledge of how to implement PLM resides in the heads of individuals who lack the incentives to share their experiences (Rangan et al., 2005). Experiences drawn from this case can be transferred to other PLM implementation projects through a thorough description of the project. Therefore, the case can be used to validate and extend existing PLM implementation guideline sets. 
Finally, with the PLM implementation guidelines, it is possible to conduct systematic studies of the execution of PLM implementation projects. However, comparisons with other cases are needed in order to provide evidence for or against the hypothesis that better application of relevant PLM implementation guidelines leads to better project outcomes. We aim to present the results from such a comparison in the near future.

\section{Acknowledgements}

This work was carried out within the Sustainable Production Initiative and the Production Area of Advance at Chalmers University of Technology in Gothenburg, Sweden. The support is gratefully acknowledged. We would also like to express our highest gratitude to all interviewees and reviewers who have participated in the study.

\section{References}

Batenburg, R., Helms, R. and Versendaal, J. (2006) 'PLM roadmap: stepwise PLM implementation based on the concepts of maturity and alignment', International Journal of Product Lifecycle Management, Vol. 1, No. 4, pp.333-351.

Berle, A. (2006) 'PLM development and implementation at Volvo 3P, using Catia V5 and Enovia V5', Paper Presented at the 1st Nordic Conference on Product Lifecycle Management, 25-26 January, Gothenburg, Sweden.

Bitzer, M., Eigner, M. and Vielhaber, M. (2008) 'Impacts of design process characteristics on the selection of PLM architectures', Paper presented at the DESIGN 2008, 10th International Design Conference, 19-22 May, Dubrovnik, Croatia.

Bokinge, M. (2011) 'PLM implementation guidelines', Technical report [unpublished], 1 March, Chalmers University of Technology, Gothenburg, Sweden.

Bokinge, M. and Malmqvist, J. (2011) 'Challenging requirements management issues in PLM implementation - findings from a retrospective case study', Paper presented at the 8th International Conference on Product Lifecycle Management, 11-13 July, Eindhoven, Netherlands.

Brown, C. and Vessey, I. (2003) 'Managing the next wave of enterprise systems: leveraging lessons from ERP', MIS Quarterly Executive, Vol. 2, No. 1, pp.65-77.

Chadha, B. and Welsh, J. (2000) 'Architecture concepts for simulation-based acquisition of complex systems', Paper presented at the 2000 Summer Computer Simulation Conference, 16-20 July, Vancouver, Canada.

CIMdata (2002) Product Lifecycle Management, CIMdata, Inc., Ann Arbor, Michigan.

Garetti, M., Terzi, S., Bertacci, N. and Brianza, M. (2005) 'Organisational change and knowledge management in PLM implementation', International Journal of Product Lifecycle Management, Vol. 1, No. 1, pp.43-51.

Grieves, M. (2006) Product Lifecycle Management: Driving the Next Generation of Lean Thinking, McGraw-Hill, New York.

Grönvall, J. (2009) 'Implementing PLM in practice', Lecture presented at the course PPU110 Product Lifecycle Management [unpublished], 18 September, Chalmers University of Technology, Gothenburg, Sweden.

Guba, E.G. and Lincoln, Y.S. (1989) Fourth Generation Evaluation, Sage, Newbury Park.

Hartman, N. and Miller, C. (2006) 'Examining industry perspectives related to legacy data and technology toolset implementation', Engineering Design Graphics Journal, Vol. 70, No. 3, pp.12-21. 
Hewett, A. (2009) 'Product lifecycle management (PLM): critical issues and challenges in implementation', Information Technology and Product Development, Annals of Information Systems, Vol. 5, No. 1, pp.81-105.

Illback, J. and Sholberg, J. (2000) 'Application integration in the Boeing enterprise', Paper presented at the Fourth International Enterprise Distributed Object Computing Conference (EDOC 2000), 25-28 September, Makuhari, Japan.

Jennings, M. and Rangan, R. (2004) 'Managing complex vehicle system simulation models for manufacturing system development', Journal of Computing and Information Science in Engineering, Vol. 4, No. 4, pp.372-378.

Kumar, R. and Midha, P. (2006) 'An intelligent web-based expert system for analysing a company's strategic PDM requirements', International Journal of Product Lifecycle Management, Vol. 1, No. 3, pp.230-248.

Morandotti, D. (2007) 'PLM design and delivery models: key issues and lessons learned from projects on the field', Paper presented at the 4th International Conference on Product Lifecycle Management, 11-13 July, Bergamo, Italy.

Pikosz, P., Malmström, J. and Malmqvist, J. (1997) 'Strategies for introducing PDM systems in engineering companies', Advances in Concurrent Engineering - CE97, 20-22 August, Rochester Hills, MI, USA, pp.425-434.

Rangan, R., Rohde, S., Peak, R., Chadha, B. and Bliznakov, P. (2005) 'Streamlining product lifecycle processes: a survey of product lifecycle management implementations, directions, and challenges', Journal of Computing and Information Science in Engineering, Vol. 5, No. 3, pp.227-237.

Saaksvouri, A. and Immonen, A. (2005) Product Lifecycle Management, Springer Berlin, Heidelberg.

Schuh, G., Rozenfeld, H., Assmus, D. and Zancul, E. (2008) 'Process oriented framework to support PLM implementation', Computers in Industry, Vol. 59, Nos. 2-3, pp.210-218.

Stark, J. (2004) Product Lifecycle Management: 21st century Paradigm for Product Realisation, Springer-Verlag, New York.

Svensson, L., Brulin, G., Ellström, P-E. and Widegren, Ö. (2002) Interaktiv forskning - för utveckling av teori och praktik (Interactive Research - for Development of Theory and Practice), Arbetslivsinstitutet, in Swedish, Stockholm.

Wognum, P. and Kerssens-van Drongelen, I. (2005) 'Process and impact of product data management implementation', International Journal of Product Development, Vol. 2, Nos. 1-2, pp.5-23.

Yin, R.K. (2003) Case Study Research - Design and Methods, 3rd ed., Sage Publications, Thousand Oaks.

Zimmerman, T. (2008) 'Implementing PLM across organization - for multi-disciplinary and cross-functional product development', $\mathrm{PhD}$ thesis, Chalmers University of Technology, Gothenburg, Sweden. 